\title{
Analysis on Space accessibility of Medical Service Facilities in Central Wuhan Based on Improvement of 2SFCA
}

\author{
Yi Wang ${ }^{1, a}$, Jun $\mathrm{Shao}^{2 *}, \mathrm{Miao} \mathrm{Xu}^{3}$, Changyou $\mathrm{Wu}^{4}$ and Yibang $\mathrm{Li}^{5}$ \\ ${ }^{1}$ Urban and Rural Planning, Wuhan University of Science and Technology, Wuhan, Hubei, 430000, China \\ ${ }^{2}$ Urban and Rural Planning, Wuhan University of Science and Technology, Wuhan, Hubei, 430000, China \\ ${ }^{3}$ Urban and Rural Planning, Wuhan University of Science and Technology, Wuhan, Hubei, 430000, China \\ ${ }^{4}$ Urban and Rural Planning, Wuhan University of Science and Technology, Wuhan, Hubei , 430000, China \\ ${ }^{5}$ Urban and Rural Planning, Wuhan University of Science and Technology, Wuhan, Hubei , 430000, China
}

\begin{abstract}
Aiming at the current imperfect hierarchical diagnosis and treatment system and the unreasonable spatial distribution of medical facilities. This article introduces 2SFCA model improved by the Gaussian distance decay function and multiple stage radius to evaluate the accessibility of medical facilities in Wuhan. The results indicate that: firstly, compared with the traditional 2SFCA model, the improved 2SFCA model is more sensitive to the identification of spatial accessibility, and the transition effect of the identification results is more obvious, which can accurately reflect the spatial distribution characteristics of the accessibility of medical facilities in Wuhan. Secondly, the distribution of medical facilities in Wuhan is obvious and the main factors that affect the accessibility value in different urban areas are quite different. Thirdly, the high value areas of medical facilities in the main urban area of Wuhan are mainly concentrated within the second ring line, the transition effect between the second and third ring lines is obvious, and the low value areas are mainly concentrated outside the third ring line. In the end, the main reasons for the low accessibility of the area outside the Third Ring Road are the low service quantity and low service ability of the community health service institutions and the high traffic resistance. The research results can provide a reference for the layout and planning of medical institutions in the main urban area of Wuhan.
\end{abstract}

\section{Introduction}

Medical and health services have been an important part of social public service facilities for a long time, and the rationality and accessibility of medical facilities layout is one of the key factors of public service. With the outbreak of COVID - 19 and the further improvement of the hierarchical diagnosis and treatment system in Wuhan, residents have put forward higher requirements for the improvement of the medical service system. But at present, large hospitals are overcrowded and community hospitals are deserted, which is a true picture of patients in many big cities. $80 \%$ of the high-quality medical resources of the whole society are concentrated in big cities, and $80 \%$ of the medical resources are concentrated in several highlevel hospitals. The high concentration of medical resources is an important reason for the difficulty of medical treatment[1]. Investigative studies have shown that at least more than half of the outpatients and inpatients can divert downward on the premise of ensuring medical quality[2]. The phenomenon of patients blindly crowding in large Grade 3-A hospitals is caused by the unbalanced hierarchical allocation of medical resources in each region. Optimizing the allocation of medical resources and building a complete hierarchical medical and treatment system have become an urgent proposition for diagnosis and treatment reform.

Therefore, this paper intends to analyse the accessibility of medical facilities in the main urban area of Wuhan by using the improved 2SFCA model. Taking residential areas as the research unit, aiming at medical facilities of different levels, the improved 2SFCA model is adopted to combine the traffic network data of Wuhan and the multi-level radius data obtained from the survey. Then, apply ARCGIS software for statistical computing and visualization analysis to scientifically measure the spatial distribution of medical facilities in Wuhan city, and to provide reference for the layout and planning of medical institutions in Wuhan city.

*Jun Shao: shaojun@wust.edu.cn 


\section{Study area and data}

\subsection{Study area}

Wuhan is an important city in central China and the capital city of Hubei Province. By the end of 2020, Wuhan had 13 administrative districts under its jurisdiction. This study takes the main urban area of Wuhan City as the research scope, with a total area of $955.15 \mathrm{~km}^{2}$. There are a total of 90 street towns, including Jiangan District, Qiaokou District, Jianghan District, Hanyang District, Wuchang District, Hongshan District and Qingshan District. The general situation of the study areas is shown in Figure 1.

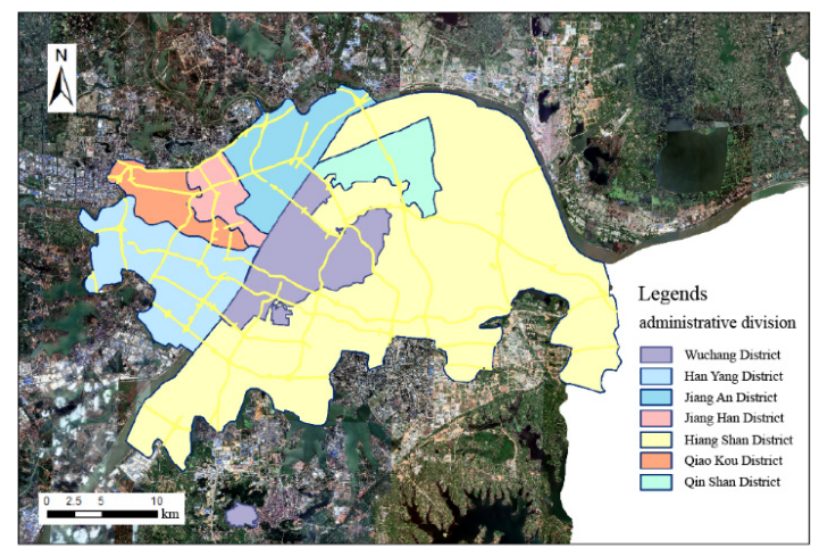

Figure 1. Research Area Overview

\subsection{Data Sources}

Taking smaller units as the research subjects can better reflect the differences in the medical resources acquired within the region. Therefore, this study takes the residential community as the minimum spatial scale to study the accessibility of medical facilities in the main urban area of Wuhan. The data mainly include:

(1) Information of medical institutions in the main urban area of Wuhan. Among the 421 medical institutions selected in this paper, there are 286 community health service institutions, 59 secondary hospitals and 76 tertiary hospitals. The data mainly came from the official website of Wuhan Health Commission Hospital and Baidu POI coordinate picking tool (Figure 2).

(2) Population data. In order to determine the demand points more accurately, the demand points here are 4934 residential areas in the main urban area of Wuhan obtained in December 2018. The geometric particle of the community is taken as the centre of mass of population distribution, and the number of households in the community is used to replace the number of population for analysis (Figure 3).

(3) Road traffic data. The road network data of the main urban area was derived from the electronic map of Wuhan in 2018, including different grades of roads such as urban expressways, expressways, national roads, provincial roads, county roads and township streets in the main urban area, and their speed attributes were assigned according to the road classification. Road speed according to Highway Engineering Design Standards (JTGB01-
2014)) [3], Code for Design of Urban Road Engineering [4], and combined with the actual traffic conditions, to determine the speed of different levels of roads (Figure 4).

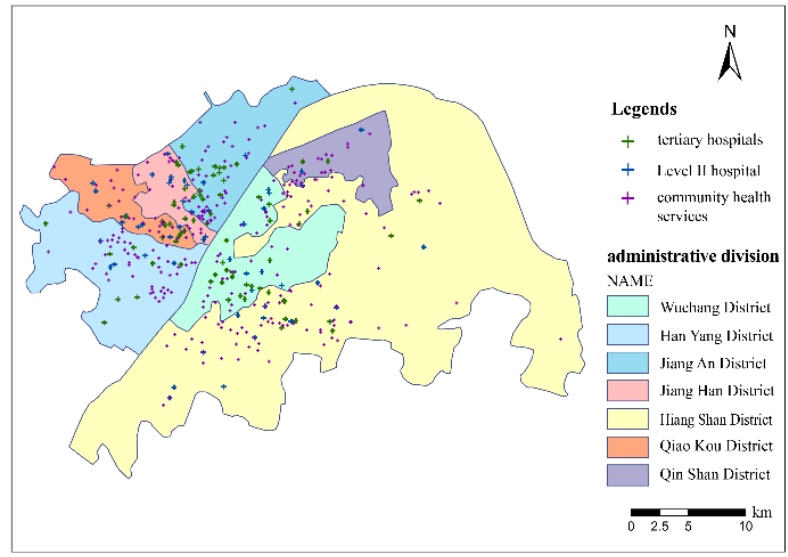

Figure 2. Distribution of Medical Points in main urban area

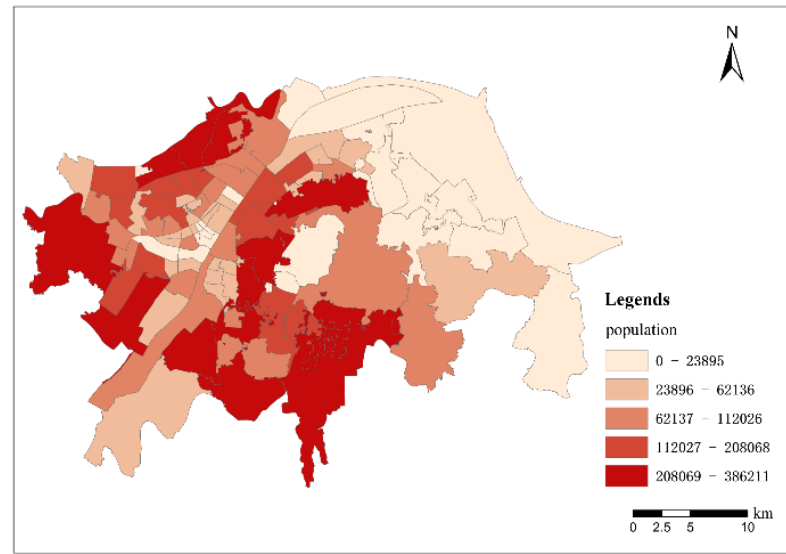

Figure 3. Main urban block population distribution

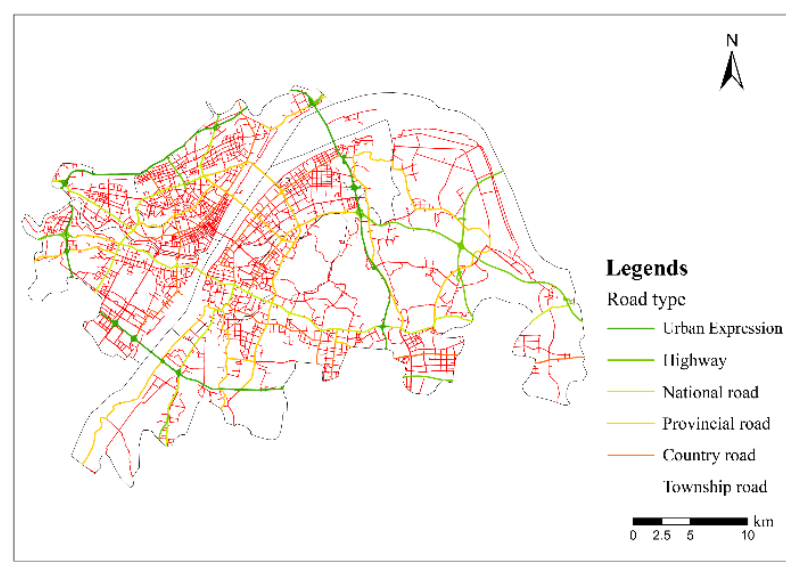

Figure 4. Main urban road classification

\subsection{Research Methods}

\subsubsection{SFCA}

1) Taking hospital $\mathrm{j}$ as the centre, determine the time threshold and search all the plot points $\mathrm{k}$ within the search radius. Then calculate the ratio of supply and demand, as shown in formula (1) 


$$
R_{j}=\frac{S_{j}}{\sum_{k \in\left\{d_{k j} \leq d_{0}\right\}} D_{k}}
$$

In the formula: $R_{j}$ is the ratio of medical service supply and demand at point $\mathrm{j} ; \mathrm{S}_{\mathrm{j}}$ is supply scale at point $\mathrm{j}$ which is expressed by the number of hospital beds in this paper. $D_{k}$ represents the demand scale of $K$ point within the search scope, which is represented by the number of residential households in the community; $d_{\mathrm{kj}}$ refers to the cost of obtaining medical services between point $\mathrm{K}$ and point $\mathrm{J}$, which is usually expressed by distance or time, time is used in this paper; $\mathrm{d}_{0}$ is the search radius.

2) From the centre of cell point $i$, search for all medical points $j$ within the search radius, then the accessibility $\mathrm{A}_{i}^{\mathrm{F}}$ at Settlement $i$ is obtained by summing up the services $R$ provided by all medical points, as shown in formula (2)

$$
A_{i}^{F}=\sum_{j \in\left\{d_{i j} \leq d_{0}\right\}} R_{j}=\sum_{j \in\left\{d_{i j} \leq d_{0}\right\}} \frac{S_{j}}{\sum_{k \in\left\{d_{k j} \leq d_{0}\right\}} D_{k}}
$$

In the formula: $A_{i}{ }^{F}$ is the accessibility of medical services at cell point $i ; d_{i j}$ is the time cost of obtaining medical services between points $i$ and $j$.

\subsubsection{Improved 2SFCA}

The traditional 2SFCA fully considers the balance between the supply and demand points and the resident competition for the limited medical resources, but ignores the impact of the hierarchical scale of the medical points on the residents' medical care conduct. Generally speaking, the larger the grade scale, the more perfect the medical facilities, the farther they can attract residents; however, the smaller the scale, the weaker the technology, and the less likely it is to attract distant residents. In addition, within the search radius, with the increase of distance, the residents' travel willingness will also be reduced, and accessibility has a law of attenuation with the distance. Based on this, Many scholars improve the 2SFCA model by extending the search radius and the introduction of the distance attenuation function. Wenli Yang[5]al. improved the 2SFCA model by referring to the shortest time of Baidu API planning service to set the multi-level radius and introducing the power function attenuation function in the accessibility study of downtown Shanghai. Li Fu[6] et al. adopted the method of field questionnaire survey to set multi-level radius and used Gaussian distance attenuation function to measure the accessibility of medical facilities in Beipei District of Chongqing. Jiangxia Yu et al. calculated the accessibility of medical facilities in the main urban area of $\mathrm{Xi}$ 'an by taking the number of medical service population as the basis and setting dynamic radius based on programming program. These extended forms make up for the deficiency of traditional single search radius to some extent, but they are also inevitably subjective and need more research and continuous improvement. For the distance attenuation, most of the extension forms are based on the distance attenuation function, and the general form is shown in formula (3) and (4). The specific extended form varies with the form of the function $\mathrm{G}\left(\mathrm{d}_{\mathrm{ij}}\right)$, mainly including the piecemeal attenuation form of gravity model kernel density form IV and Gaussian form, etc., which makes up for the shortage of treating $G\left(d_{i j}\right)$ ) as a constant in the traditional 2SFCA model.

This paper comprehensively considers the service ability of medical points, the attraction ability of different hospital scale on the residents, and the influence of distance attenuation on their travel willingness. The improved 2SFCA method adopted in this paper has three improvements compared with the traditional method. Firstly, it introduces the Gaussian distance attenuation function; secondly, using the road network data to calculate the shortest time required between the starting and ending points; in the end, through the setting of the multi-level radius and combining the actual situation of the area. The results indicate that:

For community health services, $44 \%$ of residents think that medical travel time is acceptable within $10 \sim 15$ min, and $33 \%$ think that medical travel time is acceptable within $15 \sim 20 \mathrm{~min}$. For secondary medical sites, $62 \%$ of residents consider medical travel time acceptable within $20 \sim 30 \mathrm{~min}$, and $25 \%$ believe that medical travel time is acceptable within $30 \sim 40 \mathrm{~min}$. For tertiary medical sites, $45 \%$ of residents believe that travel time is acceptable within $40 \sim 60 \mathrm{~min}$, and $20 \%$ believe that travel time is acceptable within $60 \sim 80 \mathrm{~min}$. Therefore, the maximum travel time of community health service institutions is set to the maximum travel time of $15 \mathrm{~min}$, secondary hospital to the maximum travel time of $30 \mathrm{~min}$, tertiary hospital to $60 \mathrm{~min}$.

$$
\begin{gathered}
A_{i}^{F}=\sum_{j \in\left\{d_{k j} \leq d_{0}\right\}} \frac{S_{j} \times f\left(d_{i j}\right)}{\sum_{k \in\left\{d_{k j} \leq d_{0}\right\}} D_{k} f\left(d_{i j}\right)} \\
f\left(d_{i j}\right)=\left\{\begin{array}{cc}
g\left(d_{i j}\right) & d_{i j} \leq d_{0} \\
0 & d_{i j}>d_{0}
\end{array}\right. \\
g\left(d_{i j}\right)=\frac{e^{-1 / 2 \times\left(d_{i j} / d_{0}\right)^{2}}-e^{-1 / 2}}{1-e^{-1 / 2}}
\end{gathered}
$$

In the formula: $f\left(d_{i j}\right)$ is the general form of the distance decay function; $g\left(d_{i j}\right)$ is the distance decay function within the search radius do, and the Gaussian distance decay function in this paper.

Table1. Improve the 2SFCA Model Search Thresholds

\begin{tabular}{|c|c|}
\hline Medical facilities & Search range \\
\hline Third Hospital & 60 \\
\hline Secondary Hospital & 30 \\
\hline $\begin{array}{c}\text { Community Health Services } \\
\text { Agency }\end{array}$ \\
\hline
\end{tabular}

\subsubsection{Space accessibility calculation of medical facilities}

Because the straight-line distance doesn't represent the actual distance between the two points, and the traffic 
speed of the road of different grades is also very different. In order to make the research more suitable for the actual situation, this paper takes time to characterize the travel impedance of the residents.

1) Based on the spatial location information of the residential areas and medical points, combine it with the road traffic data in ARCGIS to establish a spatial road network data set. Give different levels of roads to different speed attribute values according to Table 1, establish the OD cost matrix, and calculate the travel time of each resident to all medical points.

2) According to the established OD cost matrix, the shortest travel time of each resident to the medical point is calculated in ARCGIS, and the average of the shortest medical time consumption of each resident is $32.23 \mathrm{~min}$, so this paper sets the fixed threshold for the unimproved 2SFCA medical points to $32.23 \mathrm{~min}$. For the improved 2SFCA model, different search radii are set according to the grade scale of the medical points and the data of the field survey questionnaire. The Community Health Services are established as the $15 \mathrm{~min}$, the secondary hospitals are set to $30 \mathrm{~min}$, tertiary hospitals are set to 60 minutes.

Table2. Road speeds of different grades in Wuhan main urban area

\begin{tabular}{|c|c|}
\hline Road Type & Speed(km/h) \\
\hline Highway & 80 \\
\hline Urban Expressway & 80 \\
\hline National road & 60 \\
\hline Provincial road & 60 \\
\hline County Road & 40 \\
\hline Township streets & 20 \\
\hline
\end{tabular}

3) Taking the medical points as the starting point, all the community points falling within the fixed search radius of $32.23 \mathrm{~min}$ are obtained, and the supply and demand ratio of each medical point is calculated according to formula (1); for improvement 2SFCA, all cell points under the corresponding service threshold shall be searched according on the level of different medical points, the distance weight of the community to each medical point is calculated according to formula (4) and formula (5), and the service supply and demand ratio of each medical point is calculated.

4) Taking the community point as the starting point, calculate the sum of the supply and demand ratio of all medical points within the search threshold range; improvement 2FSCA obtains distance weight according to travel time basis formula (4) and formula (5), then calculating the weighted sum of the supply-demand ratio of the residents is worth reaching the spatial accessibility of the medical facilities of each settlement.

5) The reachability of the calculation results is assigned to each cell point and rendered in ArcGIS to obtain the reachability distribution diagram.

\section{Results and Analysis}

The spatial accessibility results of central urban medical facilities in Wuhan with traditional 2SFCA and improved 2SFCA were interpolated, and the results are shown in Figure 5 and Figure 6.

\subsection{Comparison of Traditional 2SFCA Model and improved 2SFCA Model}

Comparing Figure 5 and Figure 6, using traditional 2SFCA and improved 2SFCA, there are roughly features decreasing from east to west, from center to four sides, but there are also two differences.

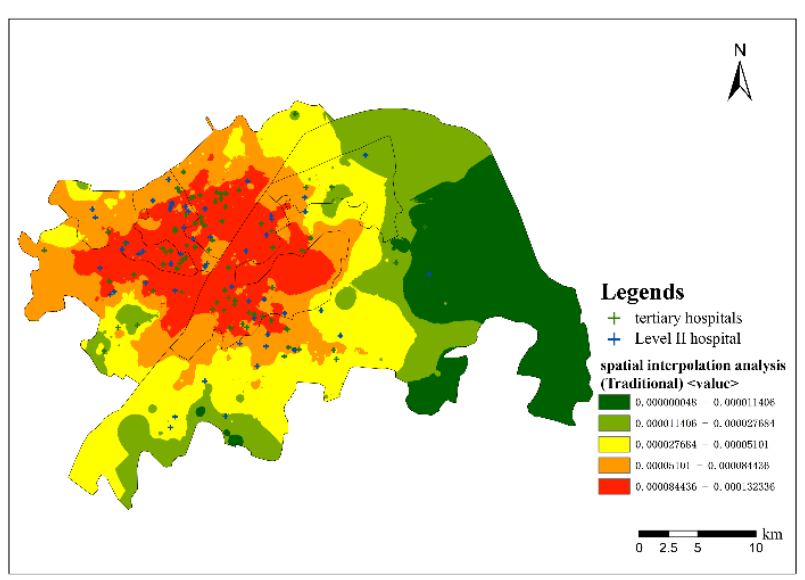

Figure 5. Traditional 2SFCA

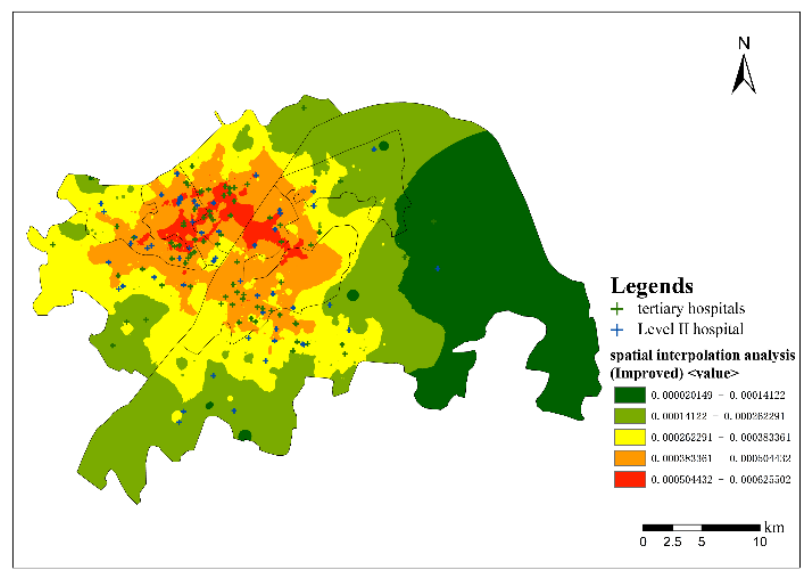

Figure 6. Improved 2SFCA

From the spatial distribution perspective, the results of traditional 2SFCA show the characteristics of two-level distribution, the circle transition result of improved 2SFCA is more significant, which are distributed at both ends of high accessibility and low accessibility. However, the circle layer transition result of improved 2SFCA is more significant, which further classifies the areas of high accessibility judged by traditional 2SFCA in a more detailed way. It is shown that the improved 2SFCA has advantages in identifying internal differences in high reachable value region. The improved 2SFCA also identifies the low accessibility of marginal areas such as Zhangjiawan Street, Qingling Town, Tianxing Town, Hongshan District, Qingjiajie Street, Jiangan District, 
Changqian Street, Gongmingcun Street, Qingshan District, which better reflects the differences in accessibility within the study area. In addition, the distribution area with the highest accessibility distribution of traditional 2SFCA is too large, some areas far from hospitals and without intensive distribution of medical points are also divided into high accessibility areas, such as Zhoutou Street in Hanyang District and Huashan Town in Donghu Development Zone ZuoLing town streets and the kanto. However, the improved 2SFCA takes into account the population scale competition for medical resources and distance attenuation factors, and the areas with the highest accessibility value only appear in the north of Wuchang District, the south of Jiangan District, the middle of Jianghan District and part of Qiaokou District, which is run through by Jianshe Avenue and Xudong Avenue. This calculation result is more consistent with the actual situation.

According to the calculation results, there is a big difference between the maximum and minimum values of the two methods, indicating that there is a big difference in the accessibility of medical facilities in the central urban area, and the distribution of medical resources is uneven. In addition, after the normalization of the calculation results of the two methods, statistical features such as the mean median standard difference are obtained, as shown in Table 3. It is found that the calculation results of the improved 2SFCA are slightly smaller than those of the traditional 2SFCA. This is because the improved 2 SFCA considering the size of the medical level and the distance between the point of supply and demand factors on the impact of accessibility value, using different search radius and add gaussian distance decay function etc, resulting in part under the traditional algorithm can reach the quantities under the improved algorithm and inaccessible or lower accessibility values, lower part of the grade Remote medical sites are excluded. It is more in line with the actual medical treatment situation, so the overall calculation result is smaller.

Table3. Comparison of the two methods

\begin{tabular}{|c|c|c|}
\hline & $\begin{array}{c}\text { Traditional } \\
\text { 2SFCA }\end{array}$ & $\begin{array}{c}\text { Improved } \\
\text { 2SFCA }\end{array}$ \\
\hline $\begin{array}{c}\text { Minimum } \\
\text { value }\end{array}$ & 0.00002 & 0.00002 \\
\hline $\begin{array}{c}\text { Maximum } \\
\text { value }\end{array}$ & 0.000726 & 0.000626 \\
\hline Average value & 0.0004675 & 0.000409 \\
\hline $\begin{array}{c}\text { Median } \\
\text { number }\end{array}$ & 0.000421 & 0.000495 \\
\hline $\begin{array}{c}\text { Standard } \\
\text { difference }\end{array}$ & 0.00011 & 0.00009 \\
\hline
\end{tabular}

To sum up, the improved 2SFCA takes into account the range attenuation factor, the grade scale factor of the medical point, and uses the Gaussian range attenuation function combined with multi-stage radius to make up for the shortcomings of the traditional 2SFCA. The calculation results are more in line with the actual situation in all aspects and are better than the traditional
2SFCA. Therefore, the following article will focus on the analysis of the calculation results of the improved 2SFCA.

\subsection{Spatial distribution of accessibility of medical facilities in the main urban area of Wuhan}

On the whole, the accessibility of medical facilities in Wuhan downtown is high, the results present the circle distribution and east-west imbalance. From the east to the west and from the centre to the periphery, the accessibility value decreases gradually, especially in the east of Hongshan District, which is the lowest area in the main urban area, while the accessibility value in the central Jianghan District of Wuchang District is higher. The distribution of medical facilities in Wuhan's main urban area can be divided into 5 circles:

1) First-class area is the highest accessibility circle, the north of Wuchang District, south of Jiangan District, central Jianghan District and Qiaokou District have the highest accessibility, belonging to the first-class area. It has obvious advantages over the surrounding area.

The perfect traffic network, good connectivity between medical sites and residents, the perfect hierarchical construction of medical institutions, reasonable spatial layout and rich overall medical resources lead the peak of medical accessibility value in this region in the overall central urban area.

2) The second-class area is the circle layer with high accessibility. Most areas in the south of Qiaokou District of Jianghan District of Wuchang District and the north of Hanyang District have better accessibility and belong to the second-class area. This area basically surrounds inside and outside the Second Ring Road of Wuhan City and occupies a large area.

However, compared with the first-class area, the population density of most blocks in this area is very high, the supply-demand balance relationship of medical resources is slightly less than that of the first-class area, but it can better meet the daily medical needs of residents.

3) The third-class area is generally accessible circle. This area is basically located between the second and the third rings in the central urban area. The regional area is large, and the population density of most blocks in the area is very high. The main problem in the three types of regions is that, on the one hand, there is a lack of hospitals in highly densely populated areas in the region, which cannot be supported by community service agencies alone; on the other hand, some areas with more hospitals in the region have the problem of unreasonable spatial distribution of supply and demand mismatch.

4) The fourth type of area is the circle layer with low accessibility. Most blocks in this area have low residential density. There are 6 level-III hospitals, 8 level-II hospitals and 26 community health service institutions in this area. The regional population density is not high, and the main reasons for the low accessibility value are the high travel impedance caused by the low road network density and the inconvenience of nearby medical treatment caused by too few community health service institutions. 
5) The five types of areas are low accessibility zones, and the areas with the worst accessibility are the eastern part of Hongshan District and a small part of Qingshan District, which are completely outside the Third Ring Road of Wuhan. Compared with other areas, the area is larger, the location is more remote, the population density is very low, and the distribution of medical points is more dispersed.

\section{Conclusion and Discussion}

Based on the basis of traditional 2SFCA, considering the influence of hospital scale and distance attenuation factors, the multi-level radius and Gaussian distance attenuation function are introduced to improve the 2SFCA model, based on GIS space analysis technology and the minimum research unit to study the accessibility of medical facilities in Wuhan, making the research results closer to reality. The research results indicate that:

1) The improved two-step mobile search method presents a more significant layer transition feature in the spatial distribution, with high sensitivity in identifying the internal differences between high and low reachability regions. In statistical features, the average and standard difference are slightly less than the traditional 2SFCA, the more uniform distribution characteristics, and the analysis found that low accessibility and general accessibility of improved 2SFCA recognition, such as Baishazhou Street, Wuchang District and Zhangjiawan Street, Hongshan District, The high accessibility area identified by traditional 2SFCA, after in-depth analysis, found that there were no secondary hospitals and tertiary hospitals in the two blocks, and the actual medical accessibility value is not high. The results of improving 2SFCA more accurately reflect the spatial distribution characteristics of accessibility of medical facilities in the central urban area of Wuhan.

2) The overall medical facilities in the central urban area of Wuhan are high, showing a trend of decreasing from center to around and from west to east.

3) The domestic research, such as Li Fu, improved the two-step mobile search method by introducing Gaussian distance decay function and multi-level service radius, and also compared the improved 2SFCA with the traditional 2SFCA results, and found that the improved statistical eigenvalues are less than the pre-improvement results, in addition, the characteristics of the spatial distribution characteristics are also inconsistent. Through comprehensive analysis, the improved results are more consistent with the actual situation and are consistent with the conclusions of this paper, it shows that the improved two-step movements can consider various factors comprehensively, and the results can more relatively accurately describe the accessibility spatial distribution of medical facilities in the study area.

The results of this paper help to understand the spatial accessibility of medical facilities in Wuhan and provide reference for the rational layout planning of medical institutions, but there are many deficiencies: firstly, still sets the maximum time threshold for residents at different levels of medical points. In the next step, the study can consider setting the sensitivity analysis of different travel time impedance to determine the threshold $\mathrm{d}_{0}$. Secondly, the study is based on the assumption that residents only seek medical treatment in the central area of Wuhan and the internal medical resources are only for residents in the study area, without considering the behavior of the surrounding area and the accessibility of the region.

\section{References:}

1. Hu X.R., Xiong W., Ren F., Mao Y., Wang G.C. (2017) Analysis of medical service capability by road network_ Taking Wuhan as an example. Surveying and GIS, 40 (11): 67 - 70.

2. Yu J.X., Zhang X.Q. (2017) Study on Medical Facilities Based on Service Radius _ Taking the main urban area of $\mathrm{Xi}$ ' an as an example. Journal of Shaanxi Normal University (Natural Science Edition), 45 (05): $78-84$.

3. Yang W.L., Chen N., Wang H.M., Yang W., Xia C.L. (2020) Study on Reachability of Medical Facilities in 2SFCA Shanghai Central Urban Area. Geographic Information World, 27 (06): 75 - 79.

4. Fu L., Wang Y., Zeng B., Mao Y., Gao M. (2019) Space Reachability Analysis of Beibei Medical Facilities Based on Improving the Two - step Mobile Search Method. Journal of Earth Information Sciences, 21 (10): 1565 - 1575.

5. Guo J.X., Liu T., Wang K.Y., Xie S.J., Zhang Y.S. (2020) Educational Resources Based on Improved Two - step Mobile Search Method _ takes the main urban area of Hangzhou as an example. Journal of Hangzhou Normal University (Natural Science Edition), 19 (03): 329 - 336.

6. Wang Q. (2019) Reachability in Shanghai Based on Improved Two - step Mobile Search. Wuhan University.

7. Liu C.C. (2019) Reachability Analysis and Layout Optimization of Medical Facilities Based on Improved 2SFCA Algorithm. Chengdu University of Technology. 